\title{
pH-Triggered Dethreading-Rethreading and Switching of Cucurbit[6]uril on Bistable [3]Pseudorotaxanes and [3]Rotaxanes
}

\author{
${\text { Dönüis Tuncel }{ }^{*[a]} \text { and Martin Katterle }}^{[b]}$
}

\begin{abstract}
A series of water-soluble [3]rotaxanes- $(n+2)$ and [3]pseudorotaxanes- $(n+2)$ with short (propyl, $n=$ 1 ) and long (dodecyl, $n=10$ ) aliphatic spacers have been prepared in high yields by a 1,3-dipolar cycloaddition reaction catalyzed by cucurbit[6]uril (CB6). The pH-triggered dethreading and rethreading of CB6 on these pseudorotaxanes was monitored by ${ }^{1} \mathrm{H}$ NMR spectroscopy. A previously reported [3] rotaxane-12 that is known to behave as a bistable molecular switch has two recognition sites for CB6, that is, the diaminotriazole moieties and the dodecyl spacer. By changing the $\mathrm{pH}$ of the system, it is possible
\end{abstract}

to observe more than one state in the shuttling process. At low $\mathrm{pH}$ values both CB6 units are located on the diaminotriazole moieties owing to an ion-dipole interaction, whereas at high $\mathrm{pH}$ values both of the CB6 units are located on the hydrophobic dodecyl spacer. Surprisingly, the CB6 units shuttle back to their initial state very slowly after reprotonation of the axle. Even after eighteen days at room temperature, only about $50 \%$ of the CB6

Keywords: cucurbituril $\cdot$ molecular switches - nanotechnology $\cdot$ rotaxanes $\cdot$ supramolecular chemistry units had relocated back onto the diaminotriazole moieties. The rate constants for the shuttling processes were measured as a function of temperature over the range from 313 to $333 \mathrm{~K}$ and the activation parameters (enthalpy, entropy, and free energy) were calculated by using the Eyring equation. The results indicate that this [3]rotaxane behaves as a kinetically controlled molecular switch. The switching properties of [3]rotaxane-3 have also been studied. However, even under extreme $\mathrm{pH}$ conditions this rotaxane has not shown any switching action, which confirms that the propyl spacer is too short to accommodate CB6 units.

\section{Introduction}

An important consideration in the construction of molecular devices for nanotechnological applications is the ability to control the amplitude and speed of motion at the molecular scale. Mechanically interlocked molecules that possess more than one recognition site, such as catenanes and rotaxanes, have great potential in this area. ${ }^{[1]}$ A rotaxane is a molecule in which a wheel-like component (also known as a ring or

[a] Dr. D. Tuncel

Chemistry Department and

UNAM-Institute of Materials Science and Nanotechnology

Bilkent University, 06800 Ankara (Turkey)

Fax: (+90)312-266-4579

E-mail: dtuncel@fen.bilkent.edu.tr

[b] Dr. M. Katterle

Fraunhofer Institute for Biomedical Engineering Am Mühlenberg 13, 14476 Potsdam (Germany)

Supporting information for this article is available on the WWW under http://www.chemeurj.org/ or from the author. macrocycle) encircles an axle-like component through noncovalent interactions. To prevent dethreading of the ring from the axle, bulky stoppers are attached at the ends of the axle. If the ends of the axle are not blocked by bulky groups, then the molecule is known as a pseudorotaxane. In these systems, the ring component can shuttle under an external stimulus (chemical, electrochemical, or photochemical) from one recognition site to another, and in doing so, they convert chemical, electrochemical, or photochemical energy into mechanical energy. To date, many elegantly designed rotaxanes and catenanes have been reported ${ }^{[2]}$ and bistable [3]rotaxanes, in particular, have great potential to act as stimuli-responsive artificial molecular muscles if carefully designed. However, there are only a few examples in the literature of rotaxanes that resemble linear artificial molecular muscles. ${ }^{[3-5]}$ In these systems, the expansion and contraction caused by the movements of the ring from one recognition site to another can be monitored by using spectroscopic methods ( ${ }^{1} \mathrm{H}$ NMR, UV-visible, fluorescence) and electrochemistry.

Cucurbit[6]uril (CB6) and its homologues (CB5, CB7, $\mathrm{CB} 8$, and $\mathrm{CB} 10)$ are examples of macrocycles that have 
been used in the preparation of rotaxanes, pseudorotaxanes, polyrotaxanes, and pseudo(polyrotaxanes). Cucurbiturils have a hydrophobic cavity and two identical hydrophilic carbonyl portals. ${ }^{[6-13]}$ CB6 binds well to protonated mono- and diaminoalkanes as a result of these structural features, mainly through ion-dipole interactions and the hydrophobic effect. ${ }^{[8]}$ Moreover, CB6 has been shown to catalyze $[3+2]$ cycloaddition reactions between suitably functionalized alkyne and azide groups to give 1,4-disubstituted triazoles, and a number of rotaxanes and polyrotaxanes have been designed and synthesized by using this catalytic effect. ${ }^{[8-11]}$ There are also a number of examples of CB6-based bistable [2]rotaxanes that behave as molecular switches. ${ }^{[12]}$

The properties of rotaxanes and pseudorotaxanes in other applications can be best exploited if their synthesis is straightforward and very efficient. Recently, we presented a bistable molecular switch prepared by an easy, high yielding CB6-catalyzed click reaction that confers these prerequisites. ${ }^{[13]}$

Herein we report the detailed synthesis and characterization of a series of [3]rotaxanes and [3]pseudorotaxanes that have either a long dodecyl or a short propyl aliphatic spacer. The $\mathrm{pH}$-dependent switching properties of these rotaxanes and pseudorotaxanes were investigated by ${ }^{1} \mathrm{H} \mathrm{NMR}$ spectroscopy. The kinetics of the acid-induced movement of CB6 units in a [3]rotaxane with a dodecyl spacer ([3]rotaxane-12) were studied and the rate constants were measured as a function of temperature in the range 313 to $333 \mathrm{~K}$. Furthermore, the activation parameters (enthalpy, entropy, and free energy) for the shuttling process were calculated by using the Eyring equation.

\section{Results and Discussion}

Synthesis of [3]pseudorotaxanes and [3]rotaxanes: We designed two types of dialkyne monomers (1 and 2; see Scheme 1) for the synthesis of the corresponding [3]pseudorotaxanes and [3] rotaxanes. We know from our previous work that the nature of the spacer (i.e., shape, size, and length) affects the catalytic ability of CB6 during [3+2] cycloaddition reactions. ${ }^{[8-10]}$ Therefore, one should carefully choose a spacer that has a lower affinity constant for CB6 than prop-2-ynylamine or azidoethylamine. It is known from the literature that CB6 can form fairly stable host-guest complexes with diaminoalkanes if the number of methylene units in the spacer is between three and eight. ${ }^{[8]}$ Accordingly, we selected propyl and dodecyl alkanes as spacers to meet this requirement. An additional reason for choosing a dodecyl alkane spacer is its ability to accommodate two CB6 units.

Rotaxanes R1 and R2 and pseudorotaxanes P1 and P2 were synthesized in high yields by following procedures described in the literature for the synthesis of similar molecules (Scheme 1). ${ }^{[11,13]}$ The products were characterized by using spectroscopic techniques $\left({ }^{1} \mathrm{H},{ }^{13} \mathrm{C}\right.$ NMR, and FTIR) and elemental analysis.

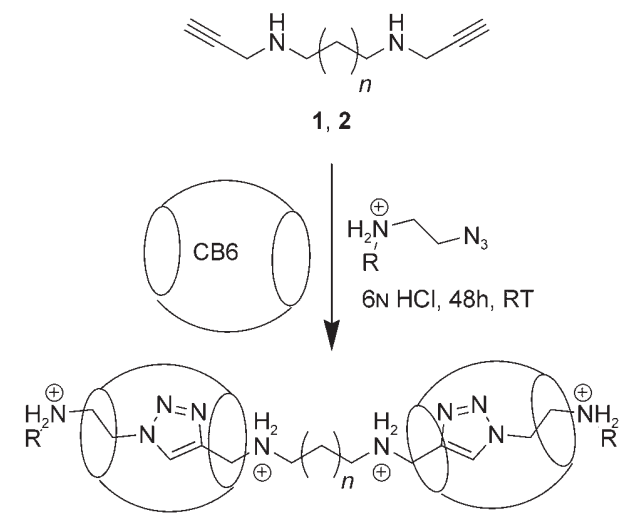

R1, R2 and P1, P2

Scheme 1. Synthesis of [3]pseudorotaxanes- $(n+2) \mathbf{P 1}(\mathrm{R}=\mathrm{H}, n=1)$ and $\mathbf{P 2}(\mathrm{R}=\mathrm{H}, n=10)$ and [3] rotaxanes- $(n+2) \mathbf{R} 1(\mathrm{R}=t \mathrm{Bu}, n=1)$ and $\mathbf{R 2}$ $(\mathrm{R}=t \mathrm{Bu}, n=10)$ from monomers $\mathbf{1}(n=1)$ and $\mathbf{2}(n=10)$. Chloride counterions have been omitted for clarity.

Characterization by MALDI-TOF mass spectrometry: The MALDI-TOF mass spectra of R1, R2, P1, and P2 were recorded by using 2,4,6-trihydroxyacetophenone (THAP) as the matrix.

The MALDI-TOF spectrum of R1 (see Figure S1 in the Supporting Information) has a series of signals. The intense signal at $\mathrm{m} / \mathrm{z} 2428.38$ has been assigned to the molecular ion of $\mathbf{R 1}$ after the loss of all counterions. There is also a signal at $\mathrm{m} / z$ 1431.94, which corresponds to an axle with only one CB6 unit. This signal is less intense than that of the molecular ion, which indicates that the energy of the laser used during sample ionization was high enough to dethread a CB6 unit from the rotaxane, even though the axle is terminated with bulky stopper groups. The signals at $m / z$ 2464.40, 2596.40 , and 2614.35 were assigned to $[M-3 \mathrm{HCl}]^{+}$, $[M+\mathrm{Na}]^{+}$, and $[M+\mathrm{K}]^{+}$, respectively.

The MALDI-TOF spectrum of P1 (see Figure S2 in the Supporting Information) has four main signals that have been assigned to the monosodium adduct of CB6 $(\mathrm{m} / \mathrm{z}$ 1019.4), an axle that has one CB6 unit $(\mathrm{m} / \mathrm{z}$ 1318.7), two CB6 units and one sodium ion $(\mathrm{m} / \mathrm{z}, 2015.7)$, and the molecular ion of $\mathbf{P 1}$ after the loss of all of its counterions $(\mathrm{m} / \mathrm{z}$ 2316.0). The signal at $\mathrm{m} / \mathrm{z} 1319.7$ shows that $\mathbf{P 1}$ has lost one CB6 unit, which suggests that dethreading occurs to a greater degree in $\mathbf{P 1}$ than in $\mathbf{R 1}$. This result is justified by the fact that dethreading of $\mathbf{P 1}$ is easier because the axle is not terminated by bulky stopper groups. The signal at $\mathrm{m} / \mathrm{z} 2015.7$ is interesting and was assigned to two CB6 units and one sodium ion. It is likely that the sodium ion connects the two CB6 units to form a dimer, and interestingly, this dimer survives both the ionization and flying processes.

The MALDI-TOF spectrum of $\mathbf{P 2}$ (see Figure S3 in the Supporting Information) has similar features to those observed for P1. However, a distinguishing feature of this spectrum is the formation of doubly charged molecular ions $(\mathrm{m} / \mathrm{z}, 1221)$, which is not common in MALDI-TOF mass spectrometry. In addition, the intensity of the signal for an 
axle with one CB6 unit is greater than the intensity of the molecular ion of P2, which indicates the dethreading of some CB6 units during the ionization process.

The MALDI-TOF spectrum of $\mathbf{R 2}^{[13]}$ (not shown) reveals small signals that were assigned to an axle of $\mathbf{R 2}$ with one CB6 unit $(\mathrm{m} / z, 1559)$ and a axle with one CB6 unit and two potassium ions $(\mathrm{m} / \mathrm{z}$ 1637). The main signal at $\mathrm{m} / \mathrm{z}, 2556$ belongs to the molecular ion of $\mathbf{R 2}$.

${ }^{1}$ H NMR spectroscopic investigation into the switching of CB6 units: [3]Rotaxanes $\mathbf{R 1}$ and $\mathbf{R 2}$ possess two different recognition sites (the diaminotriazole moieties and the dodecyl and propyl spacers) and can thereby act as molecular switches. The driving forces for the shuttling process are ion-dipole interactions and hydrophobic effects. Usually, the ion-dipole interactions are much stronger than the hydrophobic effects, but if an ion-dipole interaction is disrupted by treatment with a base, then the hydrophobic effect becomes the stronger of the two forces.

Recently, we reported the $\mathrm{pH}$-triggered switching properties of $\mathbf{R 2},^{[13]}$ as illustrated in Scheme 2. This proposed switching process is supported by the results of ${ }^{1} \mathrm{H}$ NMR spectroscopy studies (Figure 1). At low $\mathrm{pH}$ (state I; Scheme 2, Figure 1a), the CB6 units have a greater affinity for the diaminotriazole moieties than for the diaminododecamethylene moiety. The interaction between CB6 and diaminotriazole is mainly an ion-dipole interaction between the carbonyl groups of the CB6 portals and the $\mathrm{NH}_{2}{ }^{+}$ions adjacent to the dodecyl spacer and the tert-butyl groups. The CB6 units can be induced to move onto the hydrophobic dodecyl spacer if the ion-dipole interactions are disrupted by deprotonation of the ammonium ions. The resulting complex is stabilized by the hydrophobic effect and by hydrogen bonding between the $\mathrm{NH}$ groups adjacent to the dodecyl

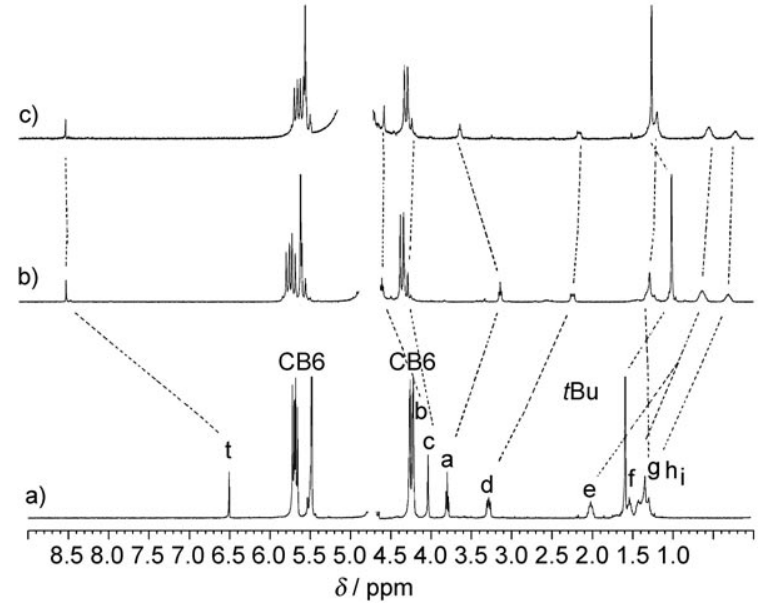

Figure 1. ${ }^{1} \mathrm{H}$ NMR $\left(400 \mathrm{MHz}, \mathrm{D}_{2} \mathrm{O}, 25^{\circ} \mathrm{C}\right)$ spectra of $\mathbf{R 2}$ a) in the absence of acid or base (state I), b) at $\mathrm{pH}>10$ after the addition of base (state II), and c) at $\mathrm{pH}<7$ after the addition of excess $\mathrm{HCl}$ (state III). See Scheme 2 for the labeling of the protons.

spacer and the carbonyl groups of CB6 (state II; Scheme 2, Figure 1b).

The addition of excess aqueous $\mathrm{HCl}$ produced some changes in the resonances of the protons adjacent to the amine groups (state III; Scheme 2, Figure 1c). For example, the signal assigned to the tert-butyl protons was shifted downfield by about $0.15 \mathrm{ppm}$, which indicated that the adjacent amine groups were protonated. However, no change was observed in the chemical shifts of the dethreaded triazole protons $(\delta=8.4 \mathrm{ppm})$ or threaded dodecyl methylene protons $(\delta=0.5-1.0 \mathrm{ppm})$, which clearly indicated that the CB6 units remained on the dodecyl spacer. In state III, the amine groups that are adjacent to the dodecyl spacer are protonated and both hydrophobic and ion-dipole interactions take effect, therefore, the activation energy required to shuttle the CB6 units back to their initial location on the diaminotriazole moieties would be high.

To find out the exact nature of the acid-induced shuttling dynamic of CB6 from the dodecyl spacer to the triazole units, we studied the kinetics of the system in more detail. At room temperature, the movement of the CB6 units is very slow and even after eighteen days, only about $50 \%$ of the CB6 units had shuttled from the dodecyl spacer back to their initial position on the diaminotriazole moieties. The kinetics of the process were then studied at
Scheme 2. The $\mathrm{pH}$ and heat-induced shuttling processes of $\mathbf{R 2}$. Chloride counterions have been omitted for clarity. Protons examined by ${ }^{1} \mathrm{H}$ NMR spectroscopy have been labeled a to $\mathrm{i}$ and $\mathrm{t}$. 
higher temperatures of $40,50,55$, and $60^{\circ} \mathrm{C}$ by using ${ }^{1} \mathrm{H}$ NMR spectroscopy. Kinetic calculations were performed by measuring the decrease in the integral area of the signal for the dethreaded triazole proton $(\delta=8.4 \mathrm{ppm})$ and the increase in the integral area of the signal for the newly threaded triazole proton $(\delta=6.5 \mathrm{ppm})$. For each spectrum, the ratio between the dethreaded and threaded protons signals was measured and the extent of shuttling at any time was calculated and plotted versus time (see the Supporting Information). First-order rate constants derived from the evaluation of ${ }^{1} \mathrm{H}$ NMR spectroscopy kinetic experiments for the acid-induced shuttling of CB6 units from state III to state I at various temperatures are shown in Table 1 and Figure 2 shows a plot of these data.

Table 1. Rate constants for the acid-induced shuttling of CB6 units in R2 from state III to state I at various temperatures, which were derived from the results of kinetic experiments. ${ }^{[a]}$

\begin{tabular}{ll}
\hline Temperature $[\mathrm{K}]$ & $k \times 10^{-3}\left[\mathrm{~s}^{-1}\right]$ \\
\hline 313 & $0.12 \pm 0.003$ \\
323 & $0.31 \pm 0.006$ \\
328 & $0.88 \pm 0.02$ \\
333 & $1.44 \pm 0.03$
\end{tabular}

[a] Monitored by using ${ }^{1} \mathrm{H}$ NMR spectroscopy.

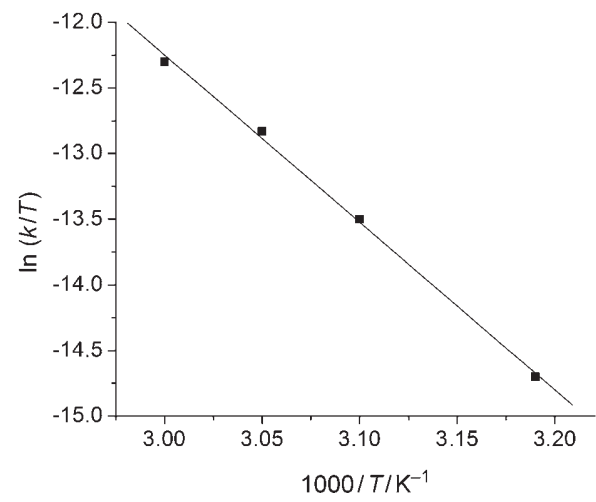

Figure 2. An Eyring plot for the shuttling of $\mathbf{R 2}$ from state III to state I.

Table 2 gives values for the free energy $\left(\Delta G^{\ddagger}\right)$, entropy $\left(\Delta S^{\ddagger}\right)$, and enthalpy $\left(\Delta H^{\ddagger}\right)$ of the acid-induced shuttling of CB6 units from state III to state I, which were derived by using the Eyring equation from the results of the ${ }^{1} \mathrm{H}$ NMR spectroscopy kinetic experiments. Our results show some similarities to those reported by Kim et al. for a kinetically

Table 2. Kinetic parameters for the acid-induced shuttling of CB6 units in $\mathbf{R 2}$ from state III to state I, which were derived from the results of kinetic experiments ${ }^{[\mathrm{a}]}$ by using the Eyring equation.

\begin{tabular}{lll}
\hline$\Delta G^{ \pm[\mathrm{b}, \mathrm{c}]}\left[\mathrm{kJ} \mathrm{mol}^{-1}\right]$ & $\Delta H^{\neq[\mathrm{b}]}\left[\mathrm{kJ} \mathrm{mol}^{-1}\right]$ & $\Delta S^{\neq[\mathrm{d}]}\left[\mathrm{J} \mathrm{mol}^{-1} \mathrm{~K}^{-1}\right]$ \\
\hline 100 & 106 & 19
\end{tabular}

[a] Monitored by using ${ }^{1} \mathrm{H}$ NMR spectroscopy. [b] Calculated at $293 \mathrm{~K}$; [c] Estimated error $<10 \%$; [d] Estimated error $>20 \%$. controlled molecular switch based on a bistable [2]rotaxane; they obtained a $\Delta G^{\neq}$value of $108 \mathrm{~kJ} \mathrm{~mol}^{-1} \cdot{ }^{[12 \mathrm{c}]}$

The switching properties of $\mathbf{R} \mathbf{1}$ were also investigated by adding varying amounts of base to a solution of $\mathbf{R} \mathbf{1}$ in $\mathrm{D}_{2} \mathrm{O}$ and recording ${ }^{1} \mathrm{H}$ NMR spectra after each addition (see the Supporting Information). Figure 3 shows the ${ }^{1} \mathrm{H}$ NMR spec-

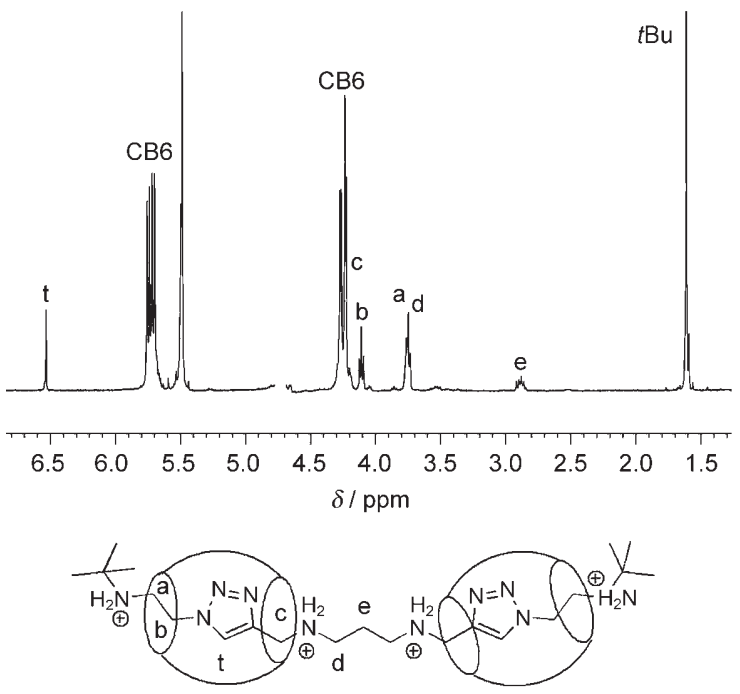

Figure 3. ${ }^{1} \mathrm{H}$ NMR (400 MHz, $\left.\mathrm{D}_{2} \mathrm{O}, 25^{\circ} \mathrm{C}\right)$ spectrum of $\mathbf{R 1}$.

trum of $\mathbf{R 1}$ in the absence of base. Several changes in the spectra were observed upon deprotonation of the ammonium ions. For example, the chemical shifts of protons adjacent to the amine groups were particularly affected and were shifted upfield by about 0.05 to $0.15 \mathrm{ppm}$. However, no change was observed in the chemical shift of the triazole proton, which indicates that the CB6 units remain on the diaminotriazole unit even after the disruption of ion-dipole interactions because the propyl spacer is too short to accommodate a CB6 unit.

${ }^{1}$ H NMR spectroscopic investigation into the dethreadingrethreading of CBs in pseudorotaxanes: When [3]pseudorotaxanes $\mathbf{P 1}$ or $\mathbf{P 2}$ are treated with aqueous $\mathrm{NaOH}$, the CB6 units are expected to either dethread from the axle and leave the diaminotriazole unit behind or shift onto the aliphatic spacer, as a result of the disruption of the ion-dipole interaction between the carbonyl groups of CB6 and the ammonium ions of the thread at higher $\mathrm{pH}$ values. Thus, CB6 units may slip off the axle and form a complex with the newly released $\mathrm{Na}^{+}$ions. To investigate which route is preferred, the $\mathrm{pH}$-triggered dethreading-rethreading of CB6 units has been monitored by ${ }^{1} \mathrm{H}$ NMR spectroscopic titration. Scheme 3 shows the proposed dethreading-rethreading process in $\mathbf{P 2}$.

Figure 4 shows the spectra of $\mathrm{pH}$-triggered dethreadingrethreading of CBs in [3]pseudorotaxanes. Before the addition of acid or base, the CB6 unit sits on the triazole moiety and as a result $\mathrm{H}_{\mathrm{t}}$ is shifted upfield (Figure $4 \mathrm{a}$ ). The addition 

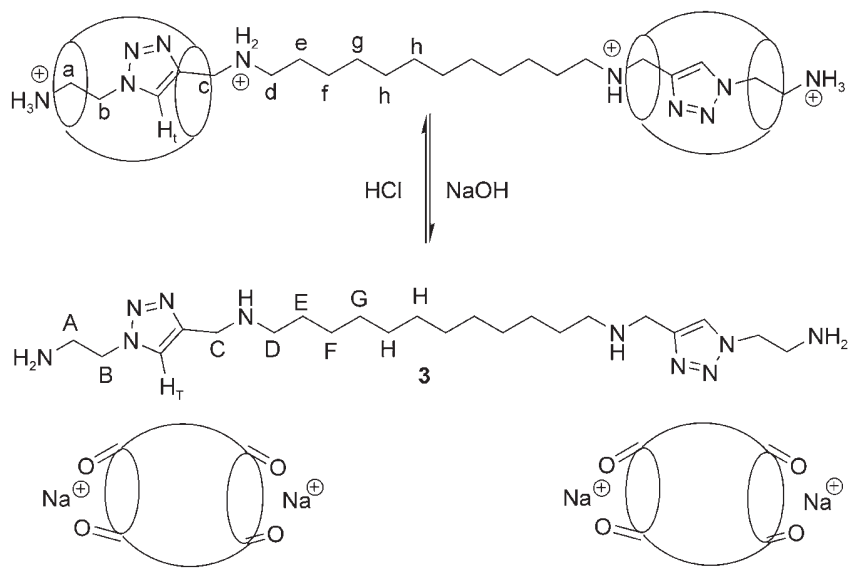

Scheme 3. Proposed pH-induced dethreading-rethreading of P2. Chloride counterions have been omitted for clarity. Protons examined by ${ }^{1} \mathrm{H}$ NMR spectroscopy have been labeled a to $\mathrm{h}, \mathrm{A}$ to $\mathrm{H}, \mathrm{t}$ and $\mathrm{T}$.

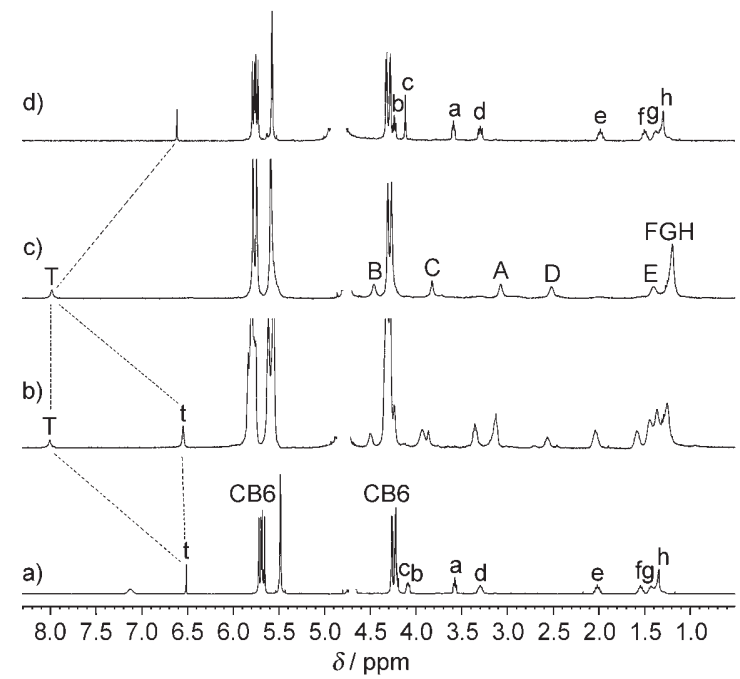

Figure 4. ${ }^{1} \mathrm{H}$ NMR $\left(400 \mathrm{MHz}, \mathrm{D}_{2} \mathrm{O}, 25^{\circ} \mathrm{C}\right)$ spectra of $\mathbf{P 2}$ a) in the absence of acid or base, b) after the addition of $\mathrm{NaOH}$ (2 equiv), c) after the addition of $\mathrm{NaOH}$ (4 equiv), and d) after the addition of $\mathrm{HCl}$ (5 equiv). See Scheme 3 for the labeling of the protons.

of $\mathrm{NaOH}$ (2 equiv) deprotonates the terminal nitrogen groups and the CB6 units start to glide. This results in two sets of signals in the spectrum, which were assigned to threaded and unthreaded triazole moieties (Figure 4b). Increasing the amount of base (4 equiv) causes all CB6 units to dethread and liberate the ditriazole axle (Figure 4c, Scheme 3). However, the addition of $\mathrm{HCl}$ (5 equiv) instantly and completely restores the initial state (Figure 4d). Thus, this $\mathrm{pH}$-induced dethreading-rethreading process is reversible.

pH-Triggered dethreading-rethreading experiments were also performed for P1, which exhibited behavior that was comparable to P2 (see Figure S4 in the Supporting Information).

\section{Conclusion}

A series of water-soluble [3]pseudorotaxanes and [3]rotaxanes with short (propyl) and long (dodecyl) aliphatic spacers were prepared in high yields through a CB6-catalyzed 1,3-dipolar cycloaddition reaction. The $\mathrm{pH}$-triggered dethreading-rethreading of CB6 units from these [3]pseudorotaxanes have been monitored by ${ }^{1} \mathrm{H}$ NMR spectroscopy. [3]Rotaxane $\mathbf{R 2}$ behaves as a bistable molecular switch with two recognition sites for CB6, namely, the diaminotriazole moieties and the dodecyl spacer. A shuttling motion can be induced by changing the $\mathrm{pH}$, and more than one state of this process was observed by using ${ }^{1} \mathrm{H}$ NMR spectroscopy. At low $\mathrm{pH}$, the $\mathrm{CB} 6$ units are located on the diaminotriazole moieties as a result of ion-dipole interactions (state I), whereas at high $\mathrm{pH}$ both $\mathrm{CB} 6$ units move onto the hydrophobic dodecyl spacer (state II). Even after treatment with acid, the CB6 units prefer to remain on the dodecyl spacer (state III) and shuttle back to state I very slowly. After eighteen days at room temperature, only about $50 \%$ of the CB6 units had relocated back onto the diaminotriazole moieties. The rate constants for this process were measured as a function of temperature over the range 313 to $333 \mathrm{~K}$ and the activation parameters for the shuttling process were calculated by using the Eyring equation. The results indicated that $\mathbf{R 2}$ behaves as a kinetically controlled molecular switch. On the other hand, [3] rotaxane $\mathbf{R 1}$ did not show any switching action, even under extreme $\mathrm{pH}$ conditions, which confirms that the propyl spacer is too short to accommodate a CB6 unit.

The kinetic studies show that after reprotonation of the axle, the rate of rethreading of CB6 units back onto the diaminotriazole moieties is quite slow in $\mathbf{R 2}$, but much faster in $\mathbf{P 1}$ and $\mathbf{P 2}$. In R2, the CB6 units must move off the dodecyl spacer, whereas in $\mathbf{P 1}$ and $\mathbf{P 2}$ the CB6 units are moving freely in solution and have formed complexes with sodium ions. In the case of $\mathbf{R 2}$, there are two binding interactions: an ion-dipole interaction between the CB6 carbonyl groups and the axle ammonium ions, and also a hydrophobic effect. In the case of $\mathbf{P 1}$ and $\mathbf{P 2}$, the only binding interaction is an ion-dipole interaction between the CB6 carbonyl groups and the sodium ions.

\section{Experimental Section}

CB6, $N, N^{\prime}$-bis(2-azidoethyl)dodecane-1,12-diamine-2 HCl, $N, N^{\prime}$-diprop-2ynyldodecane-1,12-diamine-2 $\mathrm{HCl}$ (monomer 2), $N, N^{\prime}$-diprop-2-ynylpropane-1,3-diamine-2 $\mathrm{HCl}$ (monomer 1), $N, N^{\prime}$-bis(2-azidoethyl)propane-1,3diamine $\cdot 2 \mathrm{HCl}$, tert-butylazidoethylamine $\cdot \mathrm{HCl}$ and azidoethylamine $\cdot \mathrm{HCl}$ were prepared according to the literature. ${ }^{[10-13]}$ All other reagents and solvents were of commercial reagent grade and were used without further purification, except where noted. NMR spectra were recorded by using a Bruker Avance DPX-400 MHz spectrometer. In all cases, samples were dissolved in $\mathrm{D}_{2} \mathrm{O}$ with 3-(trimethylsilyl)-1-propanesulfonic acid sodium salt (DSS) as an external standard. Mass spectra were recorded by using a Bruker Reflex II MALDI-TOF MS mass spectrometer (Bruker-Daltonik, Bremen, Germany) equipped with a nitrogen UV-laser $(\lambda=337 \mathrm{~nm})$ at $10^{-7}$ Torr and the delayed extraction mode (delay $300 \mathrm{~ns}$ ) was used by 
changing the laser intensity, if necessary. All spectra were acquired in reflector mode by using an acceleration potential of $20 \mathrm{kV}$ and are the average of 60 laser shots. The data were then transferred to a PC for further processing. THAP in $\mathrm{MeOH}\left(20 \mathrm{mg} \mathrm{m}^{-1}\right)$ was chosen as the MALDI matrix. Finally $0.1 \mu \mathrm{L}$ of this matrix was deposited on the sample plate, dried at room temperature, and analyzed.

Synthesis of R2: CB6 (342 mg, $0.342 \mathrm{mmol})$ was dissolved in $\mathrm{HCl}(6 \mathrm{M}$, $5 \mathrm{~mL}$ ) and the resulting solution was stirred for $30 \mathrm{~min}$. Monomer 2 $(60 \mathrm{mg}, 0.342 \mathrm{mmol})$ and then tert-butylazidoethylamine $\cdot \mathrm{HCl}(60 \mathrm{mg}$, $0.171 \mathrm{mmol}$ ) were added under vigorous stirring at room temperature. The resulting solution was stirred at $25^{\circ} \mathrm{C}$ for $48 \mathrm{~h}$. The solvent was removed under reduced pressure to obtain a colorless residue, which was redissolved in water $(5 \mathrm{~mL})$ and precipitated in acetone $(50 \mathrm{~mL})$. The white precipitate was collected by filtration, redissolved in water, and then filtered again through a $0.45 \mu \mathrm{m}$ filter membrane. Recrystallization was carried out by diffusing acetone vapor into the filtrate and the crystals were then collected by filtration and dried in a vacuum oven at RT for $12 \mathrm{~h}(430 \mathrm{mg}, 95 \%)$. M.p. $>300{ }^{\circ} \mathrm{C} ;{ }^{1} \mathrm{H}$ NMR $\left(400 \mathrm{MHz}, \mathrm{D}_{2} \mathrm{O}, 25^{\circ} \mathrm{C}\right)$ : $\delta=1.3-1.5(\mathrm{~m}, 8 \mathrm{H} ; f+g), 1.6\left(\mathrm{~s}, 9 \mathrm{H} ; t\right.$-butyl), $2.1(\mathrm{~m}, 2 \mathrm{H} ; e), 3.3\left(\mathrm{t},{ }^{3} J-\right.$ $(\mathrm{H}, \mathrm{H})=8.1 \mathrm{~Hz}, 2 \mathrm{H} ; d), 3.8\left(\mathrm{t},{ }^{3} J(\mathrm{H}, \mathrm{H})=6.3 \mathrm{~Hz}, 2 \mathrm{H} ; a\right), 4.1(\mathrm{~s}, 2 \mathrm{H} ; c)$, $4.15(\mathrm{t}, 2 \mathrm{H} ; b$ overlapped with $\mathrm{CB} 6), 4.2\left(\mathrm{~d},{ }^{2} J(\mathrm{H}, \mathrm{H})=15.6 \mathrm{~Hz}, 12 \mathrm{H}\right.$; CB6), 5.45 (s, $12 \mathrm{H}$; CB6), $5.94\left(\mathrm{dd},{ }^{2} J(\mathrm{H}, \mathrm{H})=15.6 \mathrm{~Hz}, 12 \mathrm{H}\right.$; CB6), $6.5 \mathrm{ppm}(\mathrm{s}, 1 \mathrm{H} ; t) ;{ }^{13} \mathrm{C}$ NMR $\left(100 \mathrm{MHz}, \mathrm{D}_{2} \mathrm{O}, 25^{\circ} \mathrm{C}\right): \delta=25.2,25.5,28.7$, 39.9, 41.8, 47.6, 49.1, 51.3 (CB6), 51.5 (CB6), 56.4, 58.6, 70.2 (CB6), 120.6 (triazole, CH), 139.0 (triazole, CR), 156.2 (CB6), 156.6 ppm (CB6); IR (KBr, pellet): $\tilde{v}=3443(\mathrm{~m}), 2927$ (w), 2353 (vw), 1735 (vs), $1473 \mathrm{~cm}^{-1}$ (vs); elemental analysis calcd (\%) for $\mathrm{C}_{102} \mathrm{H}_{136} \mathrm{Cl}_{4} \mathrm{~N}_{58} \mathrm{O}_{24} \cdot 22 \mathrm{H}_{2} \mathrm{O}$ : C 39.80, H 6.06, N 25.86; found: C 39.55, H 5.86, N 26.24 .

Synthesis of R1: Synthesized from monomer $\mathbf{1}$. The procedure was the same as that for R2 $(350 \mathrm{~g}, 90 \%)$. M.p. $>300{ }^{\circ} \mathrm{C}$; ${ }^{1} \mathrm{H} \mathrm{NMR}(400 \mathrm{MHz}$, $\left.\mathrm{D}_{2} \mathrm{O}, 25^{\circ} \mathrm{C}\right): \delta=1.6(\mathrm{~s}, 9 \mathrm{H} ; t$-butyl), $2.85-2.95(\mathrm{~m}, 1 \mathrm{H} ; e), 3.7-3.8(\mathrm{~m}$, $4 \mathrm{H} ; a+d), 3.1\left(\mathrm{t},{ }^{3} \mathrm{~J}(\mathrm{H}, \mathrm{H})=6.3 \mathrm{~Hz}, 2 \mathrm{H} ; b\right), 4.2(\mathrm{~s}, 2 \mathrm{H} ; c$ overlapped with CB6), $4.2\left(\mathrm{dd},{ }^{2} \mathrm{~J}(\mathrm{H}, \mathrm{H})=15.6 \mathrm{~Hz}, 12 \mathrm{H} ; \mathrm{CB} 6\right), 5.5(\mathrm{~s}, 12 \mathrm{H}$; CB6), 5.7 (dd, $\left.{ }^{2} J(\mathrm{H}, \mathrm{H})=15.6 \mathrm{~Hz}, 12 \mathrm{H} ; \mathrm{CB} 6\right), 6.5 \mathrm{ppm}(\mathrm{s}, 1 \mathrm{H} ; t) ;{ }^{13} \mathrm{C} \mathrm{NMR}(100 \mathrm{MHz}$, $\left.\mathrm{D}_{2} \mathrm{O}, 25^{\circ} \mathrm{C}\right): \delta=25.3,40.8,42.5,46.5,47.5,51.3$ (CB6), 51.5 (CB6), 58.6, 70.2 (CB6), 123.6 (triazole, CH), 140.0 (triazole, CR), 156.4 (CB6), $156.5 \mathrm{ppm}$ (CB6); IR (KBr, pellet): $\tilde{v}=3443$ (m), 2927 (w), 2353 (vw), 1735 (vs), $1473 \mathrm{~cm}^{-1}$ (vs); elemental analysis calcd (\%) for $\mathrm{C}_{93} \mathrm{H}_{118} \mathrm{Cl}_{4} \mathrm{~N}_{58} \mathrm{O}_{24} \cdot 21 \mathrm{H}_{2} \mathrm{O}$ ): C 37.82, H 5.46, N 27.52; found: C 38.15, $\mathrm{H}$ 5.83, N 27.18.

Synthesis of P1: Synthesized from monomer $\mathbf{1}$ and azidoethylamine $\cdot \mathrm{HCl}$. The procedure was the same as that for $\mathbf{R 2}(250 \mathrm{~g}, 90 \%)$. M.p. $>300^{\circ} \mathrm{C}$; ${ }^{1} \mathrm{H}$ NMR $\left(400 \mathrm{MHz}, \mathrm{D}_{2} \mathrm{O}, 25^{\circ} \mathrm{C}\right): \delta=2.85-2.95(\mathrm{~m}, 1 \mathrm{H} ; e), 3.5\left(\mathrm{t},{ }^{3} J-\right.$ $(\mathrm{H}, \mathrm{H})=8.1 \mathrm{~Hz}, 2 \mathrm{H} ; a), 3.8\left(\mathrm{t},{ }^{3} \mathrm{~J}(\mathrm{H}, \mathrm{H})=6.3 \mathrm{~Hz}, 2 \mathrm{H} ; d\right), 4.1(\mathrm{t}, 2 \mathrm{H} ; b)$, 4.15 (s, $2 \mathrm{H} ; c$ overlapped with CB6), $4.2\left(\mathrm{dd},{ }^{2} \mathrm{~J}(\mathrm{H}, \mathrm{H})=15.6 \mathrm{~Hz}, 12 \mathrm{H}\right.$; CB6), 5.45 (s, $12 \mathrm{H} ; \mathrm{CB} 6), 5.75\left(\mathrm{dd},{ }^{2} J(\mathrm{H}, \mathrm{H})=15.6 \mathrm{~Hz}, 12 \mathrm{H}\right.$; CB6), $6.5 \mathrm{ppm}(\mathrm{s}, 1 \mathrm{H} ; t) ;{ }^{13} \mathrm{C}$ NMR $\left(100 \mathrm{MHz}, \mathrm{D}_{2} \mathrm{O}, 25^{\circ} \mathrm{C}\right): \delta=38.4,43.5,46.5$, 47.5, 51.3 (CB6), 51.5 (CB6), 58.6, 70.2 (CB6), 121.6 (triazole, $\mathrm{CH}$ ), 139.0 (triazole, CR), 156.2 (CB6), $156.4 \mathrm{ppm}$ (CB6); IR (KBr, pellet): $\tilde{v}=3443$ (m), 2927 (w), 2353 (vw), 1735 (vs), $1473 \mathrm{~cm}^{-1}$ (vs); elemental analysis calcd (\%) for $\mathrm{C}_{85} \mathrm{H}_{102} \mathrm{Cl}_{4} \mathrm{~N}_{58} \mathrm{O}_{24} \cdot 19.5 \mathrm{H}_{2} \mathrm{O}$ : C 36.27, $\mathrm{H}$ 5.05, N 28.88; found: C 36.72, H 5.40, N 28.44.

Synthesis of P2: Synthesized from monomer $\mathbf{2}$ and azidoethylamine. $\mathrm{HCl}$. The procedure was the same as that for $\mathbf{R 2}(310 \mathrm{~g}, 95 \%)$. M.p. $>300^{\circ} \mathrm{C}$; ${ }^{1} \mathrm{H}$ NMR $\left(400 \mathrm{MHz}, \mathrm{D}_{2} \mathrm{O}, 25^{\circ} \mathrm{C}\right): \delta=1.3-1.4(\mathrm{~m}, 6 \mathrm{H} ; g+h), 1.5-1.6(\mathrm{~m}$, $2 \mathrm{H} ; f), 1.95-2.05(\mathrm{~m}, 2 \mathrm{H} ; e), 3.3\left(\mathrm{t},{ }^{3} J(\mathrm{H}, \mathrm{H})=8.1 \mathrm{~Hz}, 2 \mathrm{H} ; d\right), 3.6\left(\mathrm{t},{ }^{3} J-\right.$ $(\mathrm{H}, \mathrm{H})=6.3 \mathrm{~Hz}, 2 \mathrm{H} ; a), 4.1(\mathrm{t}, 2 \mathrm{H} ; b), 4.2(\mathrm{~s}, 2 \mathrm{H} ; c$ overlapped with CB6), $4.25\left(\mathrm{~d},{ }^{2} J(\mathrm{H}, \mathrm{H})=15.6 \mathrm{~Hz}, 12 \mathrm{H} ; \mathrm{CB} 6\right), 5.45$ (s, $12 \mathrm{H}$; CB6), 5.75 $\left(\mathrm{dd},{ }^{2} J(\mathrm{H}, \mathrm{H})=15.6 \mathrm{~Hz}, \quad 12 \mathrm{H} ; \quad \mathrm{CB} 6\right), 6.5 \mathrm{ppm} \quad(\mathrm{s}, 1 \mathrm{H} ; t) ;{ }^{13} \mathrm{C}$ NMR $\left(100 \mathrm{MHz}, \mathrm{D}_{2} \mathrm{O}, 25^{\circ} \mathrm{C}\right): \delta=25.2,25.5,28.3,28.7,38.2,41.8,47.2,49.1$, 51.2 (CB6), 51.5 (CB6), 70.2 (CB6), 120.6 (triazole, $\mathrm{CH}$ ), 139.0 (triazole, CR), 156.2 (CB6), 156.6 ppm (CB6); IR (KBr, pellet): $\tilde{v}=3443$ (m), 2927 (w), 2353 (vw), 1735 (vs), $1473 \mathrm{~cm}^{-1}$ (vs); elemental analysis calcd (\%) for $\mathrm{C}_{94} \mathrm{H}_{120} \mathrm{Cl}_{4} \mathrm{~N}_{58} \mathrm{O}_{24} \cdot 16.5 \mathrm{H}_{2} \mathrm{O}$ ): C 39.11, H 5.35, N 28.16; found: C 39.23, H 5.10, N 27.98.

Determination of the rate constants for the acid-induced shuttling of CB6 units from state III to state $I$ in R2: A solution of R2 in $\mathrm{D}_{2} \mathrm{O}$ $(3.7 \mathrm{mM})$ was prepared and an aliquot $(500 \mu \mathrm{L})$ was transferred to an
NMR tube. First, state II was obtained by adjusting the $\mathrm{pH}$ to 10 by adding an aqueous solution of $\mathrm{NaOH}$. Subsequently $\mathrm{HCl}$ was added to adjust the $\mathrm{pH}$ to $<7$ and obtain state III. The spectrum was recorded at appropriate time intervals at temperatures of $298,313,323,328,333,0$ and $338 \mathrm{~K}$, until at least $80 \%$ of the $\mathbf{R 2}$ molecules had returned to state I. Kinetic calculations were performed by measuring the decrease in the integral area of the signal for the dethreaded triazole proton $(\delta=$ $8.4 \mathrm{ppm})$ and the increase in the integral area of the signal for the rethreaded triazole proton $(\delta=6.5 \mathrm{ppm})$. For each spectrum the ratio between the signals for the dethreaded and threaded protons was measured and the extent of shuttling at any time was calculated and plotted versus time.

\section{Acknowledgements}

This research was supported by the Scientific and Technical Research Council of Turkey (TUBITAK, Grant no: 105M025).

[1] a) Molecular Catenanes, Rotaxanes, and Knots, (Eds.: J.-P. Sauvage, C. O. Dietrich-Buchecker), Wiley-VCH, Weinheim, 1999; b) special issue on molecular machines, see: Acc. Chem. Res. 2001, 34, 409, guest-edited by J. F. Stoddart; c) E. R. Kay, D. A. Leigh, Angew. Chem. 2007, 119, 72; Angew. Chem. Int. Ed. 2007, 46, 72; d) W. R. Browne, B. L. Feringa, Nat. Nanotechnol. 2006, 1, 25.

[2] a) S. A. Nepogodiev, J. F. Stoddart, Chem. Rev. 1998, 98, 1959; b) J. D. Badjic, V. Balzani, A. Credi, S. Silvi, J. F. Stoddart, Science 2004, 303, 1847; c) V. Balzani, A. Credi, F. M. Raymo, J. F. Stoddart, Angew. Chem. 2000, 112, 342; Angew. Chem. Int. Ed. 2000, 39, 334; d) A. G. Cheetham, M. G. Hutchings, T. D. W. Claridge, H. L. Anderson, Angew. Chem. 2006, 118, 1626; Angew. Chem. Int. Ed. 2006, 45, 1596; e) D. S. Marlin, D. A. Leigh, A. M. Z. Slawin, Angew. Chem. 2006, 118, 83; Angew. Chem. Int. Ed. 2006, 45, 77.

[3] Y. Liu, A. F. Flood, P. A. Bonvallet, S. A. Vignon, B. H. Northrop, H.-R. Tseng, J. O. Jeppesen, T. J. Huang, B. Brough, M. Baller, S. Magonov, S. D. Solares, W. A. Goddard, C.-M. Ho, J. F. Stoddart, J. Am. Chem. Soc. 2005, 127, 9745.

[4] M. C. Jimenez, C. Dietrich-Buchecker, J.-P. Sauvage, Angew. Chem. 2000, 112, 3422; Angew. Chem. Int. Ed. 2000, 39, 3254.

[5] a) M. C. Jimenez-Molero, C. Dietrich-Buchecker, J.-P. Sauvage, Chem. Commun. 2003, 1613; b) S. Bonnet, J.-P. Collin, M. Koizumi, P. Moban, J.-P. Sauvage, Adv. Mater. 2006, 18, 1239.

[6] W. L. Mock in Comprehensive Supramolecular Chemistry, Vol. 2 (Ed.: F. Vögtle) Pergamon, Oxford, 1996, p. 477.

[7] a) K. Kim. , Chem. Soc. Rev. 2002, 31, 96; b) Yamaguchi, K. Kim, J. Am. Chem. Soc. 2000, 122, 540; c) K. Kim, N. Selvapalam, Y. H. Ko, K. M. Park, D. Kim, J. Kim, Chem. Soc. Rev. 2007, 36, 267; d) J. Lagona, P. Mukhopadhyay, S. Chakrabarti, L. Isaacs, Angew. Chem. 2005, 117, 4922; Angew. Chem. Int. Ed. 2005, 44, 4844; e) A. Day, A. P. Arnold, R. J. Blanch, B. Snushall, J. Org. Chem. 2001, 66, 8094; f) S. Liu, P. Y. Zavalij, Y.-F. Lam, L. Isaacs, J. Am. Chem. Soc. 2007, 129, 11232; g) C.-F. Ke, S. Hou, H.-Y. Zhang, Y. Liu, K. Yang, X.-Z. Feng, Chem. Commun. 2007, 3374; h) C. Meschke, H.-J. Buschmann, E. Schollmeyer, Polymer 1998, 40, 945.

[8] a) W. L. Mock, N.-Y. Shih, J. Org. Chem. 1986, 51, 4440; b) W. L. Mock, N.-Y. Shih, J. Am. Chem. Soc. 1988, 110, 4706; c) W. L. Mock, Top. Curr. Chem. 1995, 175, 1.

[9] a) D. Tuncel, J. H. G. Steinke, Chem. Commun. 1999, 1509; b) D. Tuncel, J. H. G. Steinke, Chem. Commun. 2001, 253; c) D. Tuncel, J. H. G. Steinke, Chem. Commun. 2002, 496; d) D. Tuncel, J. H. G. Steinke, Macromolecules 2004, 37, 288.

[10] D. Tuncel, H. B. Tiftik, B. Salih, J. Mater. Chem. 2006, 16, 3291.

[11] D. Tuncel, N. Cindir, Ü. Koldemir, J. Inclusion Phenom. Macrocyclic Chem. 2006, 55, 373.

[12] a) W. L. Mock, J. Pierpont, J. Chem. Soc. Chem. Commun. 1990, 1509 ; b) S. I. Jun, J. W. Lee, S. Sakamoto, K. Yamaguchi, K. Kim, Tetrahedron Lett. 2000, 41, 471; c) J. W. Lee, K. Kim, K. Kim, Chem. 
Commun. 2001, 1042; d) K. Kim, W. S. Jeon, J.-K. Kang, J. W. Lee, S. Y. Jon, T. Kim, K. Kim, Angew. Chem. 2003, 115, 2395; Angew. Chem. Int. Ed. 2003, 42, 2293; e) J. W. Lee, K. Kim, S. W. Choi, Y. H. Ko, S. Sakamoto, K. Yamaguchi, K. Kim, Chem. Commun. 2002, 2692; f) Y. J. Jeon, P. K. Bharadwaj, S. W. Choi, J. W. Lee, K. Kim, Angew. Chem. 2002, 114, 4654; Angew. Chem. Int. Ed. 2002, 41, 4474; g) V. Sindelar, S. Silvi, A. E. Kaifer, Chem. Commun. 2006,
2185; h) V. Sindelar, S. Silvi, S. E. Parker, D. Sobransingh, A. E. Kaifer, Adv. Func. Mater. 2007, 17, 694; i) L. Yuan, R. Wang, D. M. Macartney, J. Org. Chem. 2007, 72, 4539.

[13] D. Tuncel, Ö. Özsar, H. B. Tiftik, B. Salih, Chem. Commun. 2007, 1369.

Received: December 19, 2007 Published online: March 17, 2008 